\title{
Insight on Blind Channel Estimation of MIMO-OSTBC Using Higher Order Statistics with Kalman Filter (HOS-KF)
}

\author{
${ }^{1}$ Department of E\&TC, Dr. D.Y. Patil Institute of Technology, Pimpri, Pune-411018 \\ 1,2 Army Institute of Technology Pune-411015 \\ Email:*renukabhndari6@gmail.com
}

\section{Received: $19^{\text {th }}$ Feb 2018, Accepted: $17^{\text {th }}$ March 2018, Published: $30^{\text {th }}$ June 2018}

\begin{abstract}
A Blind channel estimation of Multiple-Input Multiple-Output-Orthogonal Space Time Block Code (MIMO-OSTBC), using Higher Order Statistics with Kalman Filter (HOS-KF), is proposed. It offers better performance, improvement in spectral efficiency and moderate computational complexity. This paper focuses on channel estimation without CSI information for fast time varying MIMO systems. It adds advantages of HOS as well as of KF. Minimization of cost function obtained by higher order kurtosis is done by Kalman filter. KF estimates the value after each iteration and then update it. Matlab simulation used to evaluate the channel estimation. Performance parameters, Bit Error Rate (BER) and Mean Square Error (MES) versus Signal to Noise Ratio (SNR) are plotted. Simulation results with different modulation schemes like M-QAM and M-PSK depict that proposed method outperforms as compare to traditional Independent Component analysis (ICA) and KF method in terms of BER, MES. Spectral efficiency also improves as no Channel State Information (CSI) is needed for proposed estimation, as it is needed in KF. This all good results we got at the cost of moderate computational complexity. This is the first research of HOS and KF based channel impulse response estimation, for the time varying velocity of the receiver in an OSTBC-MIMO system. All the performance criteria BER, MES \& spectral efficiency are improved in proposed HOS- KF method.
\end{abstract}

Keywords: Channel Estimation, MIMO OSTBC, HOS, KF, CSI , ICA

\section{Introduction}

In wireless communication, conjunction with multiple-input multiple output (MIMO) systems, high data rate and quality of transmission demands are increasing significantly. To achieve these requirements of communication, MIMO technique deploys multiple antennas at both the transmitter and receiver to get the diversity and multiplexing gains [1][2]. Signal travels from transmitter to receiver takes different paths. Because of reflection, diffraction and scattering, communication suffers multipath fading. When signal reaches to receiving antenna, it is a composite signal of all multipath signals [3]. The multipath signals arrive at the receiver side have different delays and amplitudes which affect overall signal estimation as well as reliability of the system. Space-time Block Codes (STBC) is a method that receiver usually employed into systems to improve the reliability of data transmission using multiple antennas[4].Advantages of MIMO systems include, Beam forming, Spatial Multiplexing and Spatial Diversity[5]. Spatial Diversity can be achieved with MIMO -STBC system. MIMO with STBC shows improved performance in fading channels. Tarokh et al.[6], suggested Space-Time Coding (STBC) and transmitter diversity with two transmitter antennas with maximum likelihood receiver presented by Alamouti [7], After that Orthogonal Space-Time Coding (OSTBC) and Quasi-Orthogonal STBC (QOSTBC) schemes with more than two antennas are studied in[8][9]. Space-Time Codes (STCs) have been extensively and fruitfully initiated in cellular communications together with native space networks. A host of coding schemes have been kickstarted in the world that embody orthogonal STBC[10] , quasi-orthogonal STBC[11] and non-orthogonal STBCs.

With the evolution of wireless communication system, the limitation of channel and signal estimation and their effects are increasing. These both areas are also having research objectives for MIMO wireless communication system. Signal detection is an important task of communication applications like cognitive radio, software defined for defense communication, monitoring and surveillance of spectrum, electronic warfare, etc. In literature many techniques are available for Signal detection of SingleInput Single- Output (SISO) systems [12]. Now with the era of multiple-input systems, research has been shifted on detection of signal for multiple-input scenarios.

The overall performance of the MIMO- STBC can be further enhanced if a better channel estimation is involved in the system. Various methods were 
proposed in past for the improvement of signal estimation efficiency based on reference information using training based, adaptive, blind or semi blind approaches. Further enhancements are still on to improve the efficiency with minimum time convergence. It is pertinent to note that the Maximum Likelihood [13], based receivers are able to achieve the better performance with the highest diversity and small bit error ratio (BER) though it requires the highly accurate detection mechanism and its complexity is very high. In this regard, Zero Forcing (ZF) receivers and Minimum Mean Square Error receivers belong to the family of linear receivers[14][15]. Training-based methods provide either high level performance or low complexity at the cost of bandwidth efficiency [15]. On the other hand, the blind method applies the statistics of receiving signals to derive the channel information [16]. Blind estimation or semi blind techniques are computationally complex than the training based estimation, but the later origins spectral inefficiency by insertion of channel state information as training sequences along with actual transmit data. Recently, many researches are going on the techniques which do not require CSI at the receiver; e.g., blind or semi blind channel estimation [17][18][19]. Blind methods are basically classified between two categories, Second Order Statistics (SOS) \& Higher Order Statistics (HOS). Many SOS blind channel estimation methods assume that channel is invariant over STBC blocks. Thus, these SOS blind estimators are not suitable for time varying channel or quasi static channel.

In [16], generalized HOS blind channel estimation is present in literature to cover the simplest way for broader class of general STBCs. This method is useful for non-cooperative environment also because no modification is needed in transmitter.

The Kalman filtrations are been developed and used in various models of communications because of its simple coding and estimation performance. In literature many methods available for MIMO channel estimation using Kalman filter (KF)[20], Extended Kalman filter (EKF)[21]. Kalman filter requires some measurement information like channel state information (CSI), to estimate channel. A. Fereidountabar, et all [22], presented a channel estimation method for MISO \& MIMO using the Kalman filter \& STBC codes. Their simulation results based on time variant Rayleigh distributed channels. BPSK and QPSK modulation have been used.

In this paper, to assist Kalman filter, instead of CSI, HOS based [16], channel estimation technique, is used to estimate the channel. Kalman filter, together with
HOS assures improvement in BER performance without any loss in the available spectrum.

Remaining part of this paper is organized as follows: System model is described in section 2. Section 3 presents mathematical modeling of channel estimator. Simulation results are presented in section 4 and in section 5 , conclusion is given.

\section{System Model}

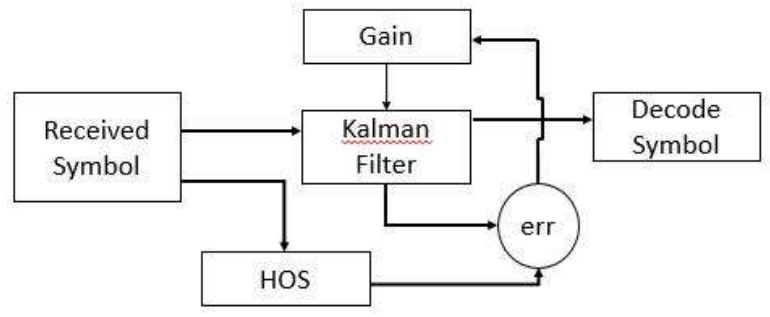

Fig. 1. Block Diagram of Proposed HOS-KF Method

Fig. 1 shows block diagram of proposed HOS-KF channel estimation. MIMO OSTBC receiver receives transmitted symbols. In the wireless MIMO-OSTBC communication system, the received signal consists of linear mixture of the transmitted data plus channel noise. The task of the blind HOS block is to distinct this linear mixture into its components without CSI. These components can be used to determine the parameters which can be then used for the estimation of channel. CSI is not needed for it and that is the beauty of blind methods. In proposed system HOS block performs minimization of higher order (kurtosis) based cost function of Zero forcing equalized signal. Simultaneously Kalman Filter first predicts the state equation and covariance matrix and generates the output. Kalman gain updates the values after each iteration, from its measurement to fit the measured data. It is obtained by the error signal which will be produced by the higher order statistic and predicted value of KF from its measurement to fit the measured data. After the channel estimation demodulation will be performed according to the modulation used at the transmitter side which is not shown here, because Fig.1 demonstrates mainly blocks for the proposed channel estimation method.

\section{$3 \quad$ Mathematical Modeling}

Notations for our discussion are as follow: Boldface lower case letters represent column vectors rep and matrices are consists of boldface uppercase letters. The Hermitian, complex conjugate and the matrix transpose, are denoted by $(\cdot) \mathrm{H},(.)^{*},(\cdot)$ T respectively; $\mathrm{N}$ XN Identity matrix is denoted by IN.

Re, Im represents real \& imaginary part of the signal respectively. 
Consider OSTBC-MIMO systems with $\mathrm{M}$ transmit and $\mathrm{N}$ receive antennas and $\mathrm{N}>\mathrm{M}$. For detection, OSTBC should be known to receiver. Let us assume the channel to be quasi static Rayleigh channel of order N X M complex channel matrix denoted by $\mathrm{H}$. Representing the complex received data by $\mathrm{Y}$. Additive white Gaussian noise is presented by $\mathrm{C}$, having zero means and some noise power. If OSTBC encoder generates nsb symbol and duration of it is lns, then using nsb symbol it creates block matrix of M X lns and this can be written as[23]

$$
\mathrm{B}_{\mathrm{d}}(\mathrm{s})=\sum_{\mathrm{k}=1}^{\mathrm{n}}\left(\mathbf{A}_{\mathrm{k}} \mathrm{R}_{\mathrm{e}}\left(\mathrm{S}_{\mathrm{k}}\right)+\mathbf{A}_{\mathrm{k}+\mathrm{n}} \mathrm{I}_{\mathrm{m}}\left(\mathrm{S}_{\mathrm{k}}\right)\right)
$$

Now energy normalization done by

$$
\begin{aligned}
& \delta=\mu\left[\left(\frac{\mathrm{B}_{\mathrm{d}}(\mathrm{s}) \cdot \mathrm{B}_{\mathrm{d}}(\mathrm{s})^{\prime}}{1_{\mathrm{ns}}}\right)\right] \\
& \mathrm{B}(\mathrm{s})=\frac{\mathrm{B}_{\mathrm{d}}(\mathrm{s})}{\sqrt{\delta}}
\end{aligned}
$$

The received block (i) is $\mathrm{N} \mathrm{X} 1_{\text {ns }}$ matrix is given by[23]

$$
\mathbf{Y}_{\mathbf{i}}=\mathbf{H} \cdot \mathrm{B}(\mathrm{s})+\mathbf{C}_{\mathbf{i}}
$$

$\mathrm{Ci}$ is $\mathrm{Nx}$ lns matrix which is additive noise Our aim is a to estimate $\mathrm{H}$ from $\mathrm{Yi}$ We can express $\mathrm{H}$ by[16]

$$
\mathbf{H}=\frac{1}{\sqrt{1_{\mathrm{c}}}} * \mathbf{U} * \mathrm{v}^{1 / 2} * \mathbf{W}
$$

Where $\mathrm{W}$ is $\mathrm{M} X \mathrm{M}$ full rank unitary matrix $\mathrm{v}=$ Real entries diagonal matrix of size M X M $\mathrm{U}$ is N X M matrix

To estimate channel and recover transmitted symbol, value of $\mathrm{W}$ should be known. In blind context, no information is present at receiver side. We can evaluate $\mathrm{W}$ by maximizing statistical independence by it's Kurtosis, denoted as K[16]

$$
\begin{aligned}
& \mathbf{W}=\left\{\min J(w)=\sum_{\mathrm{k}=1}^{\mathrm{n}} \mathrm{K}\left[\begin{array}{c}
\wedge(\mathrm{i}) \\
\mathrm{s}_{\mathrm{k}}
\end{array}\right]\right\} \\
& \text { Subject to } \mathbf{W W}^{\mathrm{H}}=\mathbf{I}_{\mathrm{nt}} \\
& \mathrm{J}(\mathrm{w})=\text { real value cost function }
\end{aligned}
$$

Gradient cost function $\mathrm{J}(\mathrm{w})$ is $\mathrm{M} \mathrm{XM}$ matrix[24]

$$
\begin{aligned}
& \tau_{\mathrm{W}}=\frac{\mathrm{d} \mathrm{J}(\mathbf{W})}{\mathrm{d} \mathbf{W}^{*}} \\
& \frac{\mathrm{dJ}(\mathbf{W})}{\mathrm{d}^{*}}=\left(\frac{\mathrm{dJ}(\mathbf{W})}{2 \mathrm{dR}_{\mathrm{e}}(\mathbf{W})}+\frac{\mathrm{dJ}(\mathbf{W})}{2 \mathrm{dI}_{\mathrm{m}}(\mathbf{W})}\right) \\
& \text { Compute descent gradient } \left.[16]^{\text {d d }}\right)
\end{aligned}
$$

$$
\mathrm{Z}=-\tau_{\mathrm{W}}
$$

$$
\begin{aligned}
& \mathbf{W}_{\mathrm{g}}=\mathbf{W}+\mathrm{Z} \mu \\
& \text { Where } \\
& \mu=1
\end{aligned}
$$

$$
\mathbf{W}_{\text {new }}=\mathbf{W}_{\mathrm{g}} *\left(\mathbf{W}_{\mathrm{g}} \mathrm{H} * \mathbf{W}_{\mathrm{g}}\right)^{-\frac{1}{2}}
$$

In our proposed method, Kalman filter estimates the value of $\mathrm{W}$ at each iteration and then updates that value from its measurement to fit the measured data. Constrained regression using Kalman filter is used. Kalman filter gives a linear, unbiased and minimum error variance recursive algorithm for the purpose to optimize the unknown channel estimation. From Kalman filter, we tend to observe that if value cost function is uncertain, co-variance matrix can decrease slowly only with small value that means measurement contributes very little to reducing the optimization problem. Kalman filter works in two steps Predictor and Corrector. In first step we do prior estimation of channel[25].

$$
\mathrm{W}_{\mathrm{k}}=\mathrm{QW}_{\mathrm{k}-1}+\mathrm{Ru}_{\mathrm{k}}+\mathrm{e}_{\mathrm{k}-1}
$$

$\mathrm{Q}$ is a state matrix and $\mathrm{R}$ is a control input matrix with $\mathrm{u}_{\mathrm{k}}$ control vector. $\mathrm{e}_{\mathrm{k}-1}$ is processing noise. Then prior error covariance is given by

$$
\mathrm{p}_{\mathrm{k}}=Q \mathrm{Qp}_{\mathrm{k}-1} \mathrm{p}^{\mathrm{T}}+\mathrm{D}
$$

D is process noise covariance matrix. Now using this predictive step we can correct error using measurement update. Kalman gain is given by

$\mathrm{K}_{\mathrm{k}}=\mathrm{p}_{\mathrm{k}} \mathrm{F}^{\mathrm{T}}\left(\mathrm{F} \mathrm{p} \mathrm{p}_{\mathrm{k}} \mathrm{F}^{\mathrm{T}}+\mathrm{T}\right)^{-1}$

$\mathrm{T}$ is measurement noise and $\mathrm{F}$ is matrix. Using this kalman gain and measurement value $\mathrm{W}$, which we estimated using higher order statistics we will perform final channel estimation. 


$$
\begin{gathered}
\mathrm{W}_{\mathrm{k}+1}=\mathrm{W}_{\mathrm{k}}+\mathrm{K}_{\mathrm{k}}\left(\mathrm{W}_{\text {new }}-\mathrm{FW}_{\mathrm{k}}\right) \\
\mathrm{p}_{\mathrm{k}+1}=\left(1-\mathrm{K}_{\mathrm{k}} \mathrm{F}\right) \mathrm{p}_{\mathrm{k}}
\end{gathered}
$$

After calculation of $\mathrm{W}$ we estimate channel $\mathrm{H}$ by equation (5).

\section{$4 \quad$ Result and Discussion}

To validate and check the performance of our proposed estimation method, Monte-Carlo simulations were performed in MATLAB 2013a. Rayleigh distributed channel with additive white Gaussian noise is considered. The simulation is carried out for $3 / 4$ code rate, OSTBC4 with 5 receiver antenna and 512 transmitted blocks. M-ary Quadrature Amplitude Modulation (MQAM) and QPSK used as modulation technique. Results are compared with the normal traditional KF and ICA method. Bit Error Rate (BER) and Mean Square Error (MSE) are used as performance parameter for the technique. Complexity of $\mathrm{KF}$ estimation is less as compare to ICA. But performance of ICA is superior to KF. Hence, this trade-off between low computational complexity and good performance has given choice of different methods.

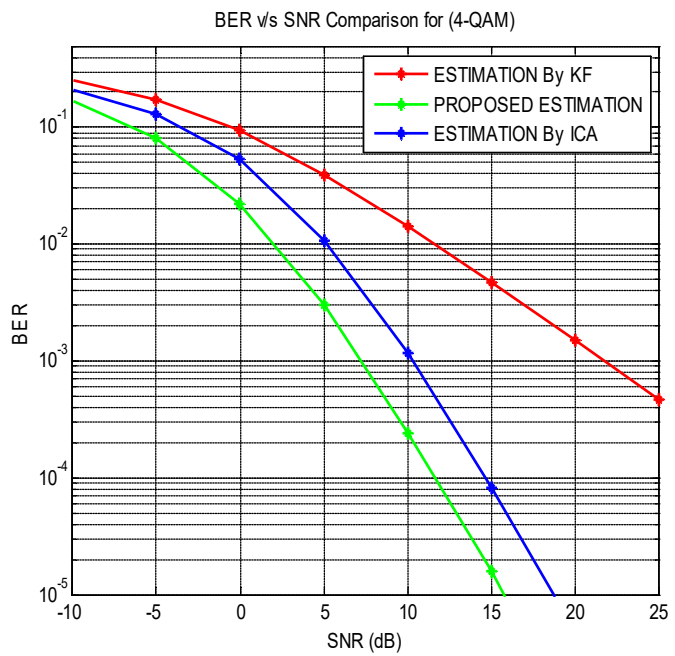

Fig.2. BER Performance of MIMO-OSTBC with 4QAM Modulation under KF, ICA \& HOS-KF

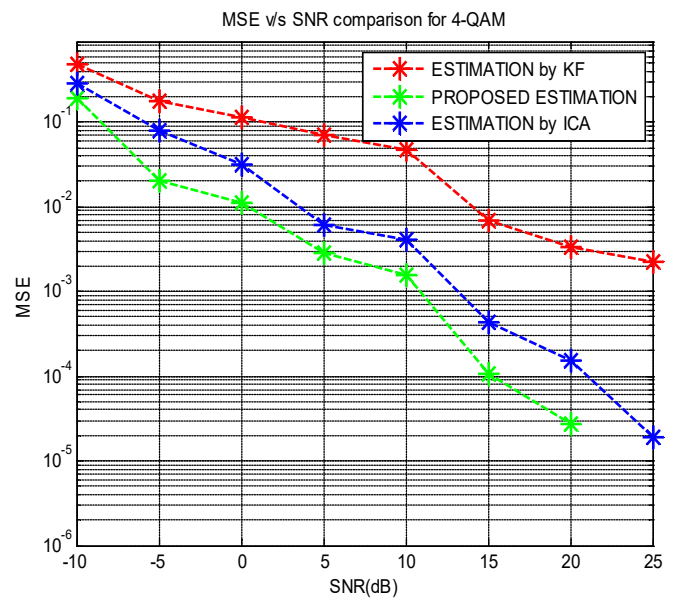

Fig.3. MSE Performance of MIMO-OSTBC with 4-QAM Modulation under KF, ICA \& HOS-KF

Fi.g 2 depicts the BER vs. SNR performance of OSTBC system with 4-QAM modulation technique. Proposed estimation HOS- KF is compared with KF and ICA. For the calculation of BER, averaging of over 150 rounds has been done. Simulation depicts that ICA outperform than KF for higher value of SNR but it is not applicable for lower SNR values. Our proposed method performs better than both KF and ICA. Our method is superior to KF because of performance and one more important point is that our method does not require any channel information.

Fig. 3 shows MSE performance of proposed estimation with KF and ICA for MIMO OSTBC system. 4- QAM modulation is used. MSE determined by using square of difference between the gains of all channels.

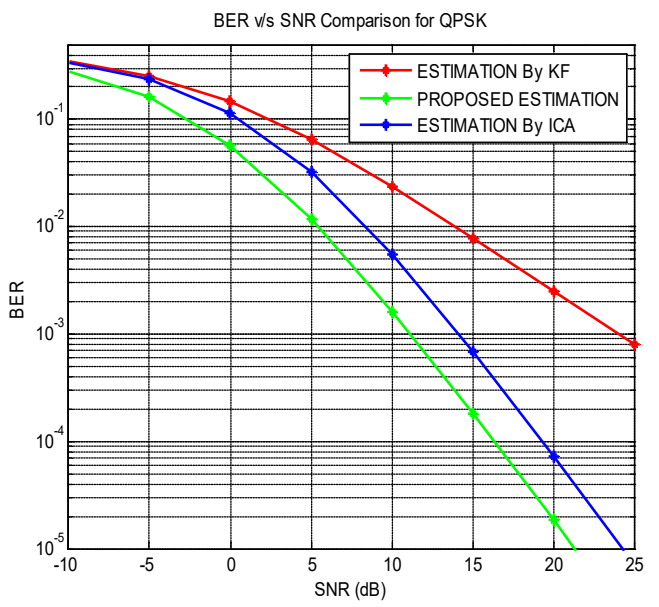

Fig.4. BER Performance of MIMO-OSTBC with QPSK Modulation under KF, ICA \& HOS-KF 


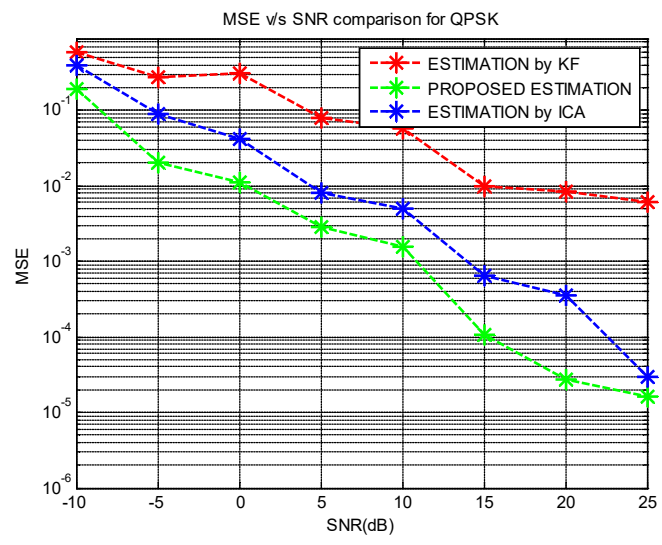

Fig.5. MSE Performance of MIMO-OSTBC with QPSK Modulation under KF, ICA \& HOS-KF

Fig.4. demonstrates the BER performance of proposed estimation with KF and ICA. QPSK modulation is used. In this scenario also HOS-KF over performed as compare to ICA and KF. Theoretically QAM gives better BER performance as compare to QPSK and it is shown in our simulation also. At the SNR value of $20 \mathrm{~dB}$ KF shows BER around $10^{-3}$, ICA shows BER value approximately $10^{-4}$ and for HOS-KF it's $10^{-5}$. Fig. 5 depicts information about MSE of the MIMOOSTBC system under KF, ICA and HOS-KF. Modulation technique used for this performance measurement was QPSK. Again proposed method shows better MSE performance as compare to other two methods. This shows that our proposed method does not depend on modulation type.

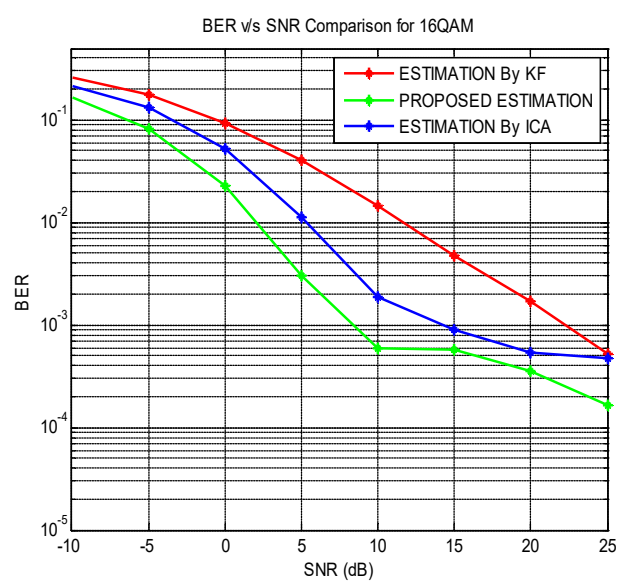

Fig.6. BER Performance of MIMO-OSTBC with 16-QAM Modulation under KF, ICA \& HOS-KF

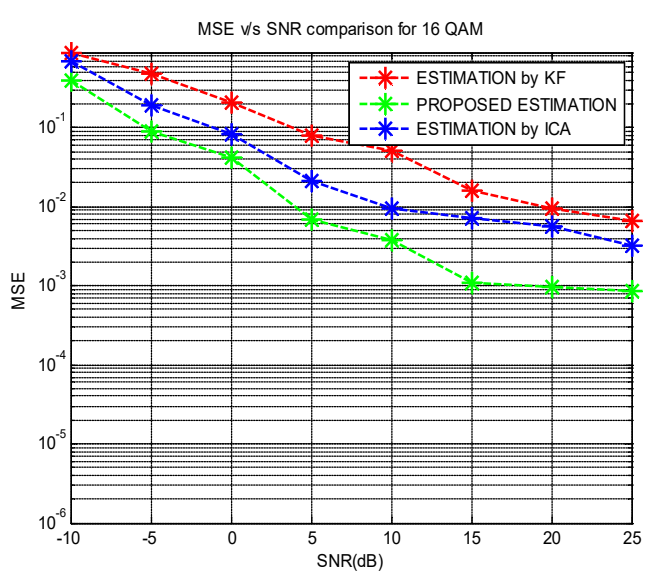

Fig.7. MSE Performance of MIMO-OSTBC with 16-QAM Modulation under KF, ICA \& HOS-KF

Fig. 6. demonstrates the BER vs. SNR plot for the MIMO- OSTBC system with 16-QAM modulation. The purpose of this plot was to verify the results for higher modulation. Simulation results validate the performance of the proposed method over KF and ICA. At $25 \mathrm{~dB}$ SNR both traditional methods show around $10^{-3}$ BER and HOS-KF shows BER around 10 ${ }^{4}$. Fig.7 depicts the MSE performance of the system for 16-QAM. As we increase modulation level MSE increases.

\section{Conclusion}

An Insight on blind channel estimation of MIMOOSTBC using HOS-KF is proposed for MIMOOSTBC system. In this paper firstly blind HOS method is used for the channel estimation by minimization of a real valued cost function and then $\mathrm{KF}$ is used to get over all performance. KF estimates the value of measurement parameter at each iteration $\&$ then update that value from its measurement to fit the measured data. Investigations of OSTBC-MIMO with blind HOS-KF conducted and simulations results are compared with traditional KF and ICA channel estimation methods. Simulation results show that less BER and MSE is achieved as compare to KF and ICA with high SNR as well as low SNR region. Additional advantage of this method is that it gives spectrum efficiency because proposed method does not require any CSI at receiver side, which is essential for other channel estimation techniques. computational complexity increased moderately. This method is best suited in performance and complexity trade off. 


\section{References}

1. G. J. Foschini, - Layered space-time architecture for wireless communication in a fading environment when using multi-element antennas, $\|$ Bell Syst. Tech. J., pp. 41-59, Autumn 1996.

2. G. J. Foschini and M. J. Gans, -On limits of wireless communications in a fading environment when using multiple antennas, $\|$ Kluwer Wireless Pers. Commun., vol. 6, pp. 311-335, Mar. 1998

3 .Rappaport T.S., Seidel S.Y. \& Singh R. (1990) 900$\mathrm{MHz}$ multipath propagation measurements for U.S. digital cellular radiotelephone. IEEE Transactions on Vehicular Technology 39(2): 132-139. DOI: http://dx.doi.org/10.1109/25.54229

4. D. Gesbert, M. Shafi, D. Shiu, P. Smith, and A. Naguib, "From theory to practice: an analysis of MIMO space-time coded wireless systems," IEEE Journal on chosen areas in Communications, vol. 21, no. 3, pp. 281-302, 2003

5. Jan Mietzner, Robert Schober, Lutz Lampe, Wolfgang H. Gerstacker, Peter A. Hoeher, MultipleAntenna Techniques for Wireless Communications A Comprehensive Literature Survey,\| IEEE communications survey and tutorials, Vol II No 2, Second quarter 2009

6. V. Tarokh, H. Jafarkhani, and A. Calderbank, - Space-time block codes from orthogonal designs, IEEE Trans. on Information Theory, vol. 45, no. 5, pp. 1456-1467, July 1999.

7. S. M. Alamouti, -A simple transmit diversity technique for wireless communications, IIEEE J. Select. Areas Commun., vol. 16, no. 8, pp. 1451-1458, Oct. 1998

8. Jafarkhani, H. (2005). Space-time coding: Theory and practice. Cambridge: Cambridge University Press

9. Tirkkonen, O., \& Hottinen, A. (2002). Squarematrix embeddable space-time block codes for the complex signal constellations. IEEE Transactions for Information Theory, 48, 384-395

10. G. Ganesan and P. Stoica, "Space-time block codes: a maximum SNR approach", IEEE Trans. Inf. Theory, Vol. 47, No. 4, pp. 1650-1656, 2001.

11. H. Jafarkhani, "A quasi-orthogonal space-time block code", IEEE Trans. Commun., Vol. 49, No. 1, pp. 1-4, 2001.

12. O. A. Dobre, A. Abdi, Y. Bar-Ness, and W. Su, "Survey of automatic modulation classification methods: classical approaches and new trends," IET Commun., vol. 1, no. 2, pp. 137-156, Apr. 2007.
13. BERENGUER, I., AND WANG, X. Space-Time coding and signal processing for MIMO communications. Journal of Computer Science and Technology 18, 6 (November 2003), 689-702.

14. R. Böhnke, D. Wübben, V. Kühn, and K. Kammeyer, "Reduced complexity MMSE detection for BLAST architectures," IEEE Global Commun. Conf., vol. 4, pp. 2258- 2262, Dec. 2003

15. Gao, F., Zeng, Y., Nallanathan, A., \& Ng, T. S. (2008). Robust subspace blind channel estimation for cyclic prefixed MIMO OFDM systems: Algorithm, identifiability and performance analysis. IEEE Journal on Selected Areas in Communications, 26, 378-388

16. Vincent Choqueuse, Ali Mansour, Gilles Burel, Senior Member, IEEE, Ludovic Collin, and Koffi Yao, "Blind Channel Estimation for STBC Systems Using Higher-Order Statistics", IEEE Transactions on wireless communications, voL. 10, no. 2, Feb 2011.

17.Wan F, Zhu W P, Swamy M N S. "A signal perturbation free whitening-rotation-based semi-blind approach for MIMO channel estimation," IEEE Transactions on Signal Processing, Vol 57(8), pp 3154-3166, 2009

18. Y. Zeng, W. H. Lam, and T. S. Ng, "Semiblind channel estimation and equalization for MIMO spacetime coded OFDM," IEEE Trans. Circuits Syst. I, Reg. Papers, vol. 53, no. 2, pp. 463-474, Feb. 2006.

19. H. Li, "Differential space-time modulation over frequency-selective channels," IEEE Trans. Signal Process., vol. 53, no. 6, pp. 2228-2242, Jun. 2005.]

20. Christos Komninakis et al, -Multi-Input MultiOutput Fading Channel Tracking and Equalization Using Kalman Estimationll, IEEE Transactions On Signal Processing, Vol. 50, No. 5, May 2002.

21. G. Ignatius, K. V. U. Murali, N. S. Krishna, P. V. Sachin, and P. Sudheesh, "Extended Kalman Filter based estimation for fast fading MIMO channels", Proceedings of International Conference on Devices, Circuits and Systems (ICDCS), 2012, pp. 466-469

22. A. Fereidountabar, G. C. Cardarilli, L. D. Nunzio, \& R. Fazzolari, "UAV Channel Estimation with STBC in MIMO Systems," Procedia Computer Science, vol. 73, pp. 426-434, 2015

23. B. Gu, J. Liu, and Y. Yu, "Orthogonal detection of beam space time block coding using ICA," in IEEE Neural Netw. Brain, vol. 2, Beijing, China, Oct. 2005, pp. 836-840. 
Helix Vol. 8(4): 3431- 3437

24. D. Brandwood, "A complex gradient operator and its application in adaptive array theory," IEE Process., Parts F and H, vol. 130, no. 1, pp. 11-16, 1983.

25. An Introduction to the Kalman Filter, Greg Welch, Gary

Bishop

http://info.acm.org/pubs/toc/CRnotice.html 